\title{
Treatment of Chronic Lower Extremity Wound Complicated by Osteomyelitis: Using Partial Biceps Femoris Muscle Flap
}

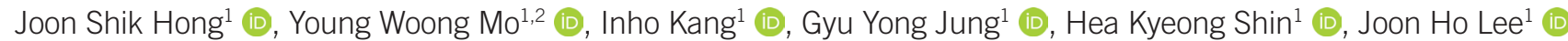 \\ ${ }^{1}$ Department of Plastic and Reconstructive Surgery, Dongguk University College of Medicine, Gyeongju; ${ }^{2}$ Department of Plastic and Reconstructive Surgery, \\ Dongguk University Graduate School of Medicine, Seoul, Korea
}

\begin{abstract}
In many cases chronic wounds in the lower extremities are extremely difficult to heal because of inadequate blood supply, and when wounds are complicated by osteomyelitis, plastic surgeons and orthopedic surgeons must join forces to facilitate treatment. A 63-year-old man with bone exposure and a peripherally indurated chronic open wound complicated by osteomyelitis of the right distal thigh was admitted to the department of orthopedic surgery at our hospital. He was referred to our department for treatment of the right side chronic open wound. We report our successful experience with a partial biceps femoris muscle transposition flap with fasciocutaneous expanded-keystone advancement flap following wound and bone debridement for the coverage of complex wounds associated with bone infection. The results show two discrete layers of muscle. In patients with chronic osteomyelitis, fasciocutaneous flaps might provide a more effective means of covering full-depth soft tissue defects without donor site morbidity, compared to free flaps.
\end{abstract}

Keywords: Hamstring muscles; Wound closure technique; Wounds and injuries

\section{Introduction}

Chronic wounds in the lower extremities are very difficult to heal due to poor circulation. Also, if a wound is complicated by osteomyelitis, it is more difficult to correct surgically; in such cases plastic and orthopedic surgeons join forces to effect treatment [1]. Some studies indicate that soft-tissue defects of the distal thigh, especially around the knee, must be treated using vascular pedicle island flaps. Most of these flaps are transferred from the back of the lower leg or from the anterior thigh, both of which receive blood supply from perforators of the popliteal artery [2-7]. We report a rare case of refractory ulceration leading to a chronic wound complicated by osteomyelitis of 32 years duration due to trauma that had been addressed surgically several times, and describe our experience of successful surgical wound care after debridement and flap coverage. The chronic wound was successfully treated with a short head of the biceps femoris (SHBF) muscle transposition flap, followed by a fasciocutaneous (FC) expanded-keystone advancement flap. These two planar flaps filled the cavity and we believe offer a suitable alternative to flap coverage of lower extremity bone exposure defects. The study was approved by the Institutional Review Board of Dongguk University Hospital (IRB No. 110757-202004-HR-04-02) and performed in accordance with the principles of the Declaration of Helsinki. The patient also provided written informed consent for the publication and the use of his images.

\section{Case Report}

Received: July 24, 2020

Revised: October 10, 2020

Accepted: October 11, 2020

\section{Corresponding author:}

Joon Ho Lee, M.D.

Department of Plastic and Reconstructive Surgery, Dongguk University College of Medicine, 87 Dongdae-ro, Gyeongju 38067, Korea

Tel: +82-54-770-1460

Fax: +82-54-770-1469

E-mail: urrussa@naver.com

This is an Open Access article distributed under the terms of the Creative Commons Attribution Non-Commercial License (https://creativecommons.org/licenses/by-nc/4.0/) which permits unrestricted non-commercial use, distribution, and reproduction in any medium, provided the original work is properly cited.

(c) 2021 Korean Wound Management Society 


\section{Case}

A 63-year-old man with a bone-exposed, peripherally indurated chronic open wound complicated by osteomyelitis at the right distal thigh was referred to the department of orthopedic surgery at our hospital for treatment of the chronic open wound. The patient had severe asthma and needed to have his wound covered under spinal anesthesia. He was referred to our department of plastic and reconstructive surgery for wound coverage.

The patient had suffered a motorcycle accident in 1988, and at the time had sustained open fractures of the right femur midshaft and underwent open reduction and internal fixation (ORIF) surgery. However, the surgical wounds became infected, and between 1988 and 2000, the wound infection was operated on at other hospitals about six times. In 2000, he underwent fracture saucerization and sequestrectomy, and received ORIF using the Ilizarov apparatus at our department of orthopedic surgery. Chronic open wounds subsequently developed and chronic osteomyelitis was diagnosed by bone biopsy. The patient was hospitalized with chronic osteomyelitis several times over the years and was treated conservatively. Unfortunately, in February 2004, he was involved in another motorcycle accident, and after conservative treatment the external fixator was removed by an orthopedic surgeon. In 2008, he was involved in a car accident and fractured at the same right femur, for which he underwent ORIF at our orthopedic surgery department. He retained an internal fixator and had open wounds, but did not attend scheduled follow-ups. Twelve years later (around December 2019), he was admitted at our department of orthopedic surgery again. The neglected wound on the right leg was open, chronicized, and indurated with much scarring, and the bone was chronically infected (Fig. 1).

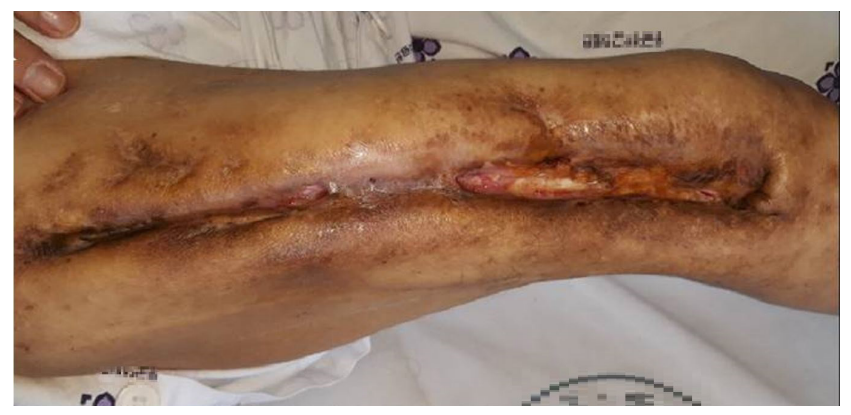

Fig. 1. Preoperative photograph. Uncontrolled chronic open wounds of the right lateral thigh of 32 years' duration. Indurated, scarred tissue of the right thigh and more severe bone-exposed wound of the distal thigh.
There was little knee function because it was fixed by the fixator from right thigh to lower leg for a long time. Flexion contracture was measured at $15^{\circ}$ and further flexion at $60^{\circ}$ just before surgery.

First of all, in order to ascertain the exact current wound condition of the lower extremity, bone biopsy and culture were performed, from which chronic osteomyelitis was confirmed. Ceftriaxone was administered intravenously for about 6 weeks, and the slough discharge was reduced, but we concluded that conservative treatment including antibiotics alone would not control the chronic osteomyelitis. As a result, we decided to debride the dirty tissues with scarring and to cover the skin defect with clean subcutaneous tissue and skin.

For surgery, the patient was placed on the operation table in the left lateral decubitus position and spinal anesthesia was performed by an anesthesiologist. Preparation was performed in the usual manner with draping and disinfection. Local anesthetic was injected (2\% lidocaine mixed with 1:100,000 epinephrine) at the site of the defect and incision site, and this was followed by compression for up to 10 minutes waiting for vasoconstriction to occur.

The right proximal wound with relatively lax surrounding skin flaps was operated on first. The peripheral indurated margin and fibrotic collagenous scar tissue of this chronic wound were debrided until clean fresh bleeding was observed. The debrided defect was then massively irrigated and meticulously hemostatized. Flaps including full-depth skin and some subcutaneous fat layers were elevated on both sides of the surgical defect and then advanced to cover the defect (Fig. 2). After insertion of a silastic drain, layer-by-layer suturing was performed to eliminate any remaining dead space.

Next, we decided to cover the chronic wound area of the bone-exposed distal thigh. Similar to the defect on the proximal wound, the peripheral indurated margin and fibrotic collagenous scarred tissue of the chronic wound of this area were debrided until clean fresh bleeding was exposed (Fig. 3). The chronically exposed distal femur bone was debrided using a burr to produce multifocal bone bleeding, and then the musculotendinous junction of the SHBF was partially cut to preserve function as much as possible. The cut muscle flap was then transposed toward the bone-exposed defect area, and the SHBF and vastus lateralis (VL) muscles were sutured to cover the dead space above exposed bone (Fig. 4). The vacant cavity over the SHBF transposition flap was packed with an expanded FC keystone advancement flap containing subcutaneous and skin layers. The keystone design was performed on the 

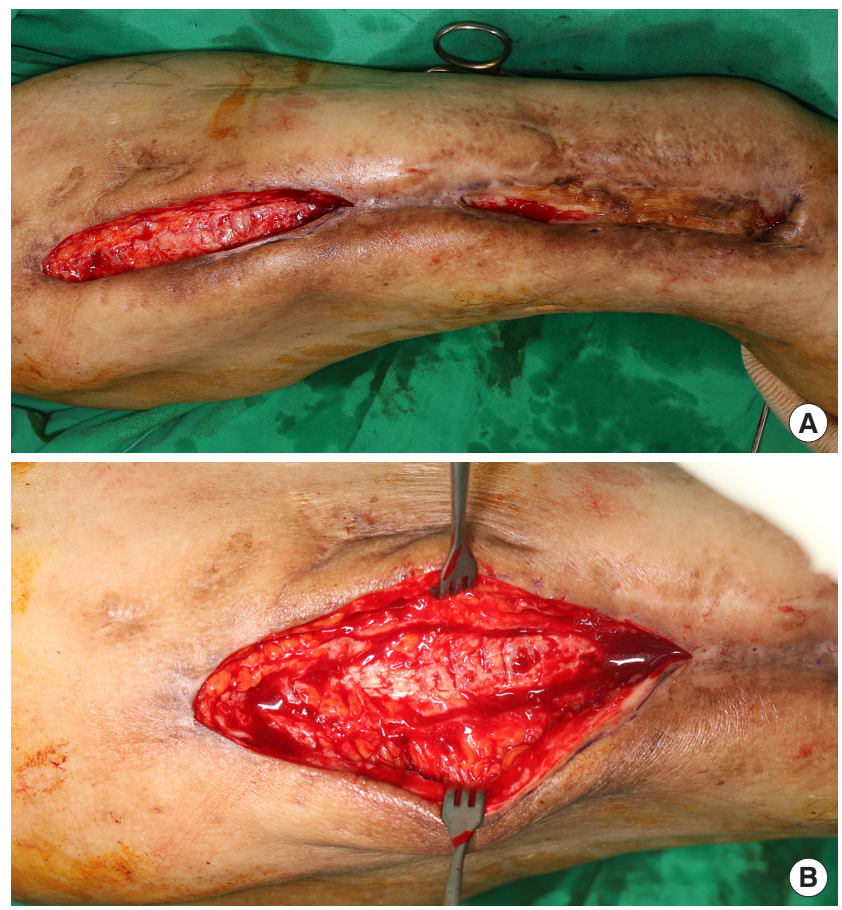

Fig. 2. Intraoperative photographs. In proximal thigh, (A) peripheral indurated scar tissue of the chronic wound was debrided. (B) Flaps were elevated on both sides of the surgical defect.

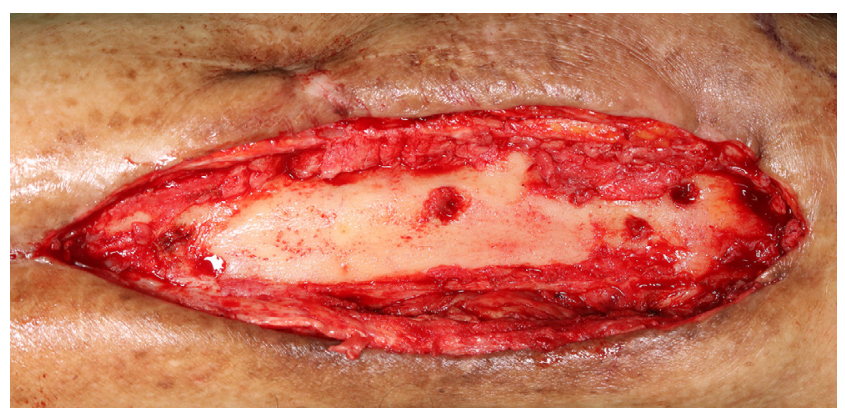

Fig. 3. Intraoperative photograph. In distal thigh, peripheral indurated scar tissue of the distal chronic thigh wound was debrided until clean fresh bleeding was exposed. The chronically exposed distal femur bone was burred to cause multifocal bone bleeding.

destructed skin over the tensor fascia lata (TFL) and a part of the VL (Fig. 5), and the FC flap was elevated (Fig. 6A). After massive irrigation and meticulous hemostasis, the keystone flap was advanced, two Hemovacs were inserted, and the wound was closed layer-by-layer (Fig. 6B).

No complications occurred after surgery, and flap circulation was satisfactory. On the 5th day after surgery, the amount of Hemovac drainage markedly decreased and no hematoma was observed, thus all silastic drains and Hemovacs were re-
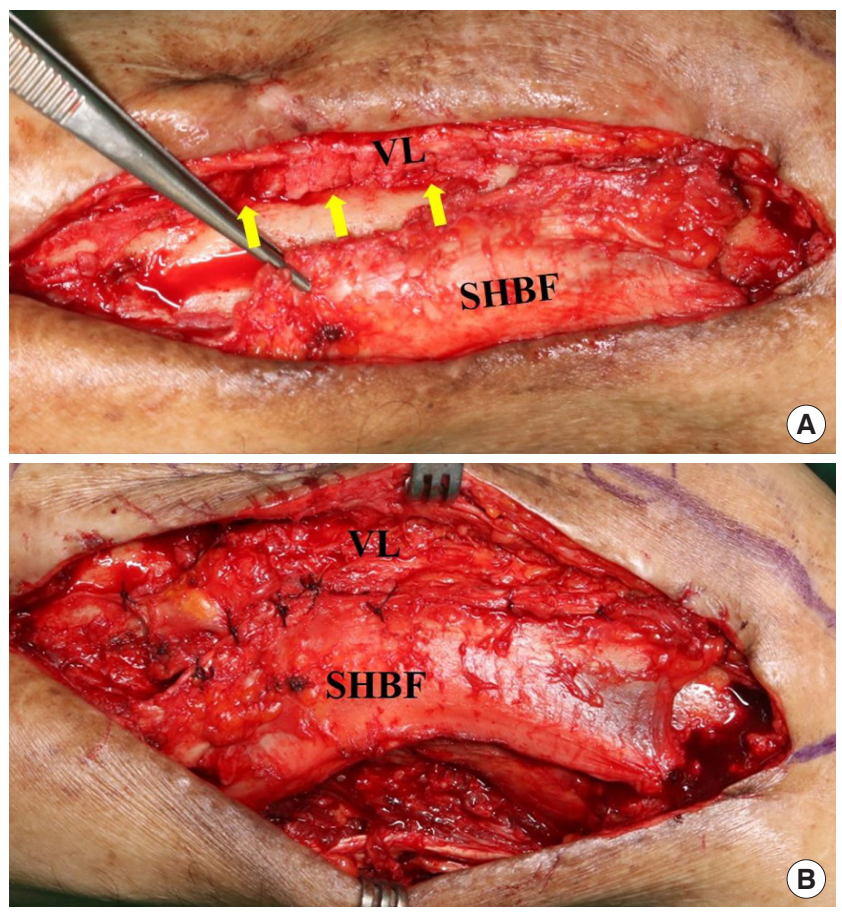

Fig. 4. Transposition of biceps femoris muscle flap. (A) The tendinous junction of the biceps femoris short head was partially cut and transposed toward the muscle layer above the bone-exposed defect of the proximal thigh to cover dead space (yellow arrows). (B) The vacant cavity over the SHBF transposition flap was packed with an expanded fasciocutaneous keystone advancement flap containing subcutaneous and skin layers. SHBF, short head of the biceps femoris; VL, vastus lateralis.

moved. On the 14th day after surgery, staples and stitches were removed, and function of the partially transposed biceps femoris was found to be preserved, thus facilitating ambulation. Four weeks after surgery, after observing the operated wound, we stopped the administration of intravenous antibiotics and decided to maintain oral antibiotics for a week after discharge. Although there was a very small skin defect, it was treated with secondary intention (Fig. 7). Our patient is undergoing rehabilitation for ambulation, and although no improvement of flexion contracture was noticeable, further flexion was better than before surgery.

\section{Discussion}

Various flaps from the posterior thigh area are commonly used to treat ischial or sacral pressure sores [8-10]. However, few reports have been issued on the transfer of a muscle flap from the posterior thigh region to the distal thigh and knee area. 

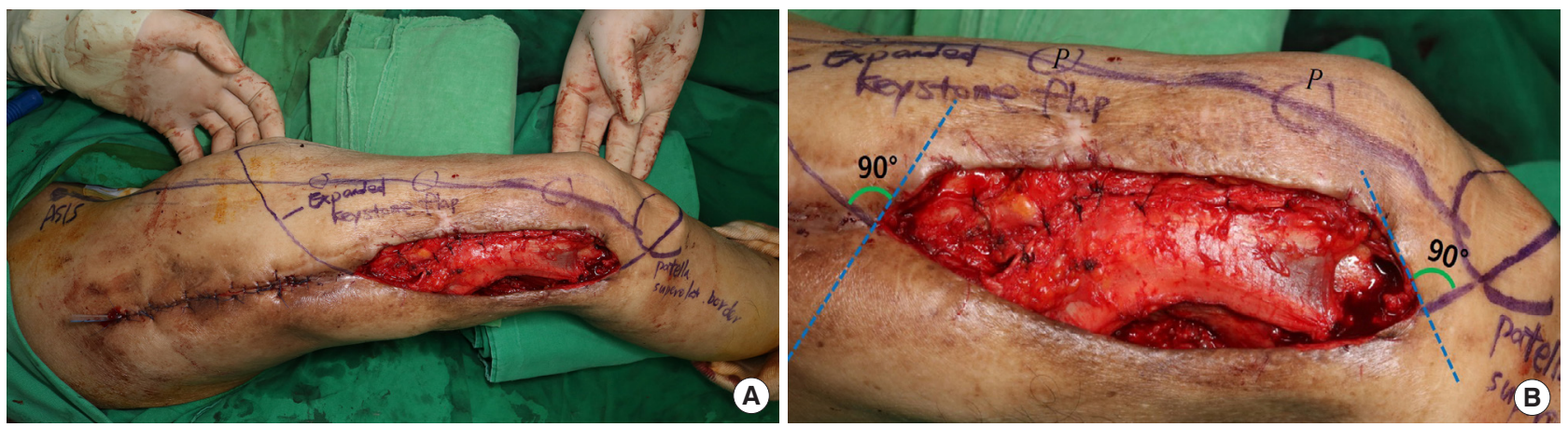

Fig. 5. Fasciocutaneous keystone advancement flap design. (A) The fasciocutaneous expanded keystone advancement flap was designed over the tensor fascia lata and vastus lateralis regions. A line was drawn from the anterior superior iliac spine (ASIS) to the lateral border of the patella, to prevent undermining medial to the line so that reliable vascular pedicles would not be injured. (B) Maximum width was noted. The side of the defect with greater tissue laxity was chosen as the flap donor area. An incision line at $90^{\circ}$ to either end of the ellipse was drawn to meet the curvilinear line of the flap outer margin. Perforators were identified by Doppler sonography on the surface of the right thigh. P, perforator.
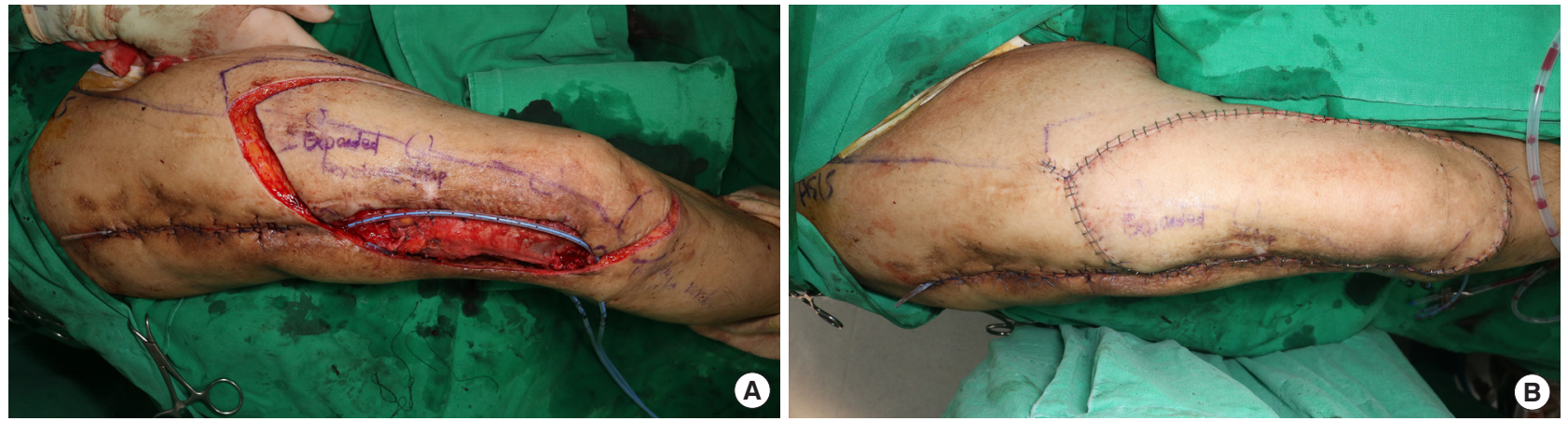

Fig. 6. Closure of the keystone flap. (A) An expanded fasciocutaneous (FC) keystone advancement flap, including subcutaneous and skin layers, was used to cover the short head of the biceps femoris transposition flap. The FC flap was undermined and elevated until the flap was mobile enough. (B) The wound was closed layer-by-layer using Vicryl 2-0, 3-0, nylon 3-0, and a surgical stapler.
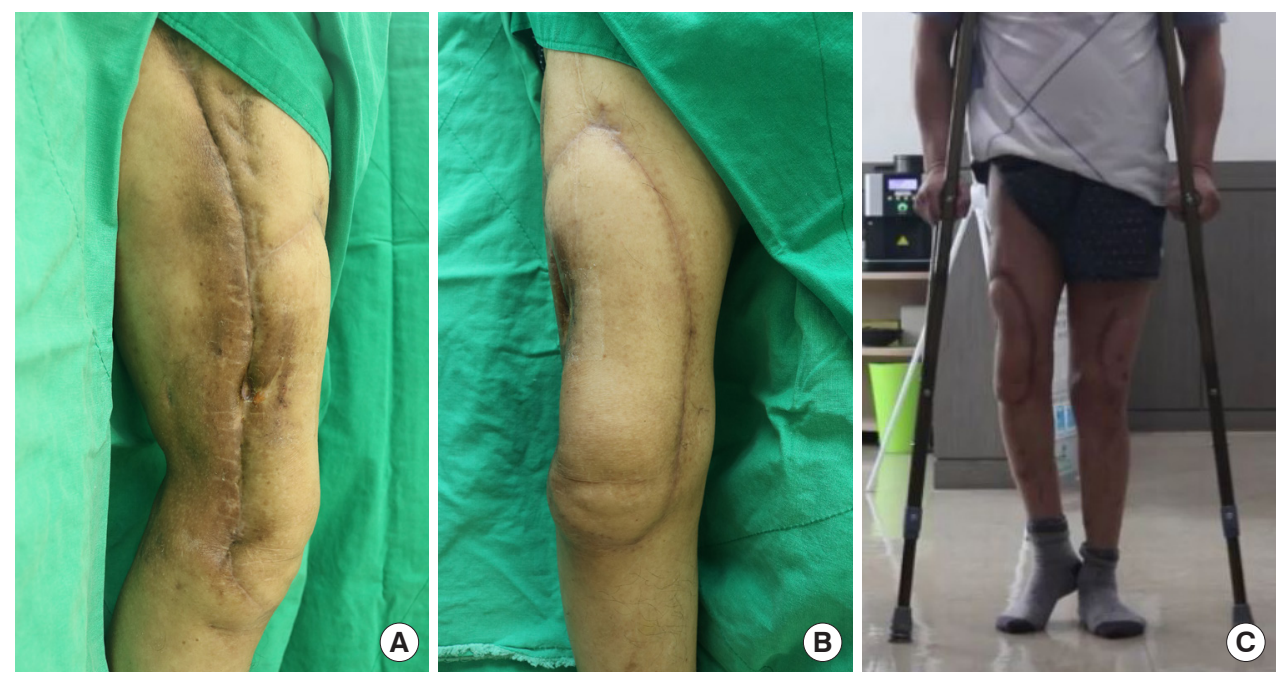

Fig. 7. Six months postoperative follow-up photographs. (A, B) There was a very small skin defect but it was treated with secondary intention. (C) The patient can ambulate using clutches. The knee function was restored slightly because the fixator was removed and short head of the biceps femoris was used partially for covering the chronic wound. 
Vascular pedicle island flap is often referred to as a means of reconstruction of soft-tissue defects around the popliteal fossa. The descending genicular artery perforator flap [11] and the reverse-flow anterolateral thigh perforator flap $[2,12]$ are typical examples. These methods are relatively reliable and safe, but a change of posture is needed during surgery. As alternatives, the medial sural artery perforator flap [5] and the reverse peroneal artery flap $[6,13]$ are available, but are likely to require a skin graft at donor sites.

Previously, musculocutaneous (MC) flaps were mainly used for distal thigh reconstruction, including sites near the knee, but we used a partial SHBF muscle flap without compromising lower limb ambulation, thus making lower leg movements more physiologic and functional. A discrete layer combination flap was produced using a FC keystone flap, which is shallower than other muscle layers of the lower extremity. In our patient, SHBF was pulled out and transposed because the right knee had little function preoperatively, which is why we chose this combination of muscle flap and FC keystone flaps. Previously used flaps were either perforator flaps or MC flaps, which required dissection from muscle to skin, undermining, and transposing, which increases complication risks. Therefore, we used two discrete layers to cut the musculotendinous junction slightly in the posterior region and the FC flap in the anterior region to maintain function of the hamstring muscle group as much as possible and to maximize ambulation.

A small-scale study demonstrated the utility of the keystone flap for the reconstruction of surgical defects. Compared with split-thickness skin grafts, keystone flaps heal with fewer complications and better aesthetics when used to reconstruct surgical defects of the lower extremities. Moreover, when compared with free tissue transfer, there is no secondary donorsite deformity. However, in order to minimize complications including wound dehiscence and infection, the operator should always consider the flap size, location and tissue laxity $[14,15]$.

To the best of our knowledge, exposure of a large bone like the femur with osteomyelitis for more than 30 years has not been previously reported. In our case, the wound was deep and extended from skin to bone, and the amount of muscle and subcutaneous tissue needed for reconstruction were considerable. In order to reconstruct this large defect while maintaining function of the lower extremity, we utilized a partial SHBF muscle transposition flap and an FC expanded-keystone flap to maximize physiologic limb ambulatory function. In our case, the TFL appeared to be absent, the VL had atro- phied to the level of a few strands of muscle fibers, and there was considerable scarring. Biceps femoris contributes to the posterolateral stability of the knee. Although the patient had poor knee joint function, we cut the musculotendinous junction of SHBF and made a flap to preserve maximum stability of the knee. After the operation, the patient's passive range of motion has not been reduced, and he found it easier to walk on crutches. The flexion of the left knee showed a slight improvement, probably owing to fixator removal and rehabilitation after the operation.

Though we had initially considered a free flap because the surgeon was more accustomed to the technique, taking into account the patient's generally poor condition and the osteomyelitis in his lower extremity, the muscle flap was preferred to the MC flap, and our choice turned out to be appropriate. All procedures were performed with spinal anesthesia, and the total time required was 2 hours and 25 minutes. This was a sufficiently short surgery considering the patient's general condition. Calf flaps are usually used for the distal thigh, but we thought it would be better to use SHBF because the fixator had been attached to the knee for several decades and the patient had little function of the knee joint.

In our patient, an SHBF muscle transposition flap and an FC expanded-keystone flap were used as a feasible reconstructive option for a substantial full depth soft-tissue and skin defect complicated by osteomyelitis of the distal thigh area around the knee. The results achieved suggest that two discrete layers of muscle and FC flaps provide a safe, reliable, and effective means of covering full-depth soft tissue defects and preserving bone integrity in patients with chronic osteomyelitis. However, one clinical case is insufficient to warrant the safety and reproducibility of the procedure. Nevertheless, we believe the described combination flap offers a reliable treatment option, especially in patients with significant full-depth damage, whether caused by trauma or iatrogenic chronic complications, to the lower extremity.

We suggest the advantages and disadvantages of this flap be studied from a statistical perspective and compared versus other methods with respect to short- and long-term complications and flap survival.

\section{Conflict of interest}

No potential conflict of interest relevant to this article was reported. 


\section{ORCID iDs}

$\begin{array}{ll}\text { Joon Shik Hong } & \text { https://orcid.org/0000-0003-3947-1435 } \\ \text { Young Woong Mo } & \text { https://orcid.org/0000-0002-4180-3139 } \\ \text { Inho Kang } & \text { https://orcid.org/0000-0002-7098-6958 } \\ \text { Gyu Yong Jung } & \text { https://orcid.org/0000-0002-0653-9766 } \\ \text { Hea Kyeong Shin } & \text { https://orcid.org/0000-0002-6248-7104 } \\ \text { Joon Ho Lee } & \text { https://orcid.org/0000-0003-0726-9881 }\end{array}$

\section{References}

1. Al-Mousawi A, Sanese G, Baljer B, et al. Use of the keystone perforator island flap in the treatment of chronic lower extremity wounds complicated by osteomyelitis. Injury 2020;51:744-9.

2. Demirseren ME, Efendioglu K, Demiralp CO, et al. Clinical experience with a reverse-flow anterolateral thigh perforator flap for the reconstruction of soft-tissue defects of the knee and proximal lower leg. J Plast Reconstr Aesthet Surg 2011;64:1613-20.

3. Feldman JJ, Cohen BE, May JW Jr. The medial gastrocnemius myocutaneous flap. Plast Reconstr Surg 1978;61:5319.

4. Hayashi A, Maruyama Y. The medial genicular artery flap. Ann Plast Surg 1990;25:174-80.

5. Xie XT, Chai YM. Medial sural artery perforator flap. Ann Plast Surg 2012;68:105-10.

6. Schneider LF, Kaplan KA, Mehrara BJ. Pedicled peroneal artery flap for popliteal fossa reconstruction. J Plast Reconstr Aesthet Surg 2014;67:282-4.

7. Tang ML, Liu XY, Ren JW, et al. The sartorius myocutane- ous island flap. Surg Radiol Anat 1993;15:259-63.

8. Hurteau JE, Bostwick J, Nahai F, et al. V-Y advancement of hamstring musculocuataneous flap for coverage of ischial pressure sores. Plast Reconstr Surg 1981;68:539-42.

9. James JH, Moir IH. The biceps femoris musculocutaneous flap in the repair of pressure sores around the hip. Plast Reconstr Surg 1980;66:736-9.

10. Tobin GR, Sanders BP, Man D, et al. The biceps femoris myocutaneous advancement flap: a useful modification for ischial pressure ulcer reconstruction. Ann Plast Surg 1981; 6:396-401.

11. Sananpanich K, Atthakomol P, Luevitoonvechkij S, et al. Anatomical variations of the saphenous and descending genicular artery perforators: cadaveric study and clinical implications for vascular flaps. Plast Reconstr Surg 2013; 131:363e-372e.

12. Yildirim S, Avci G, Akan M, et al. Anterolateral thigh flap in the treatment of postburn flexion contractures of the knee. Plast Reconstr Surg 2003;111:1630-7.

13. Ikeda K, Morishita Y, Nakatani A, et al. Total knee arthroplasty covered with pedicle peroneal flap. J Arthroplasty 1996;11:478-81.

14. Khouri JS, Egeland BM, Daily SD, et al. The keystone island flap: use in large defects of the trunk and extremities in soft-tissue reconstruction. Plast Reconstr Surg 2011;127: 1212-21.

15. Sorooshian P, King I, Dunkin C. Keystone island flaps for reconstruction following lower leg skin cancer resection: a comparison with split-thickness skin grafts. J Plast Reconstr Aesthet Surg 2019;72:513-27. 